\title{
Does education of primary care professionals promote patient self- management and improve outcomes in chronic disease? An updated systematic review
}

\author{
Claire Collins ${ }^{1 *}$, Gillian Doran ${ }^{2}$, Patricia Patton ${ }^{3}$, Roisin Fitzgerald ${ }^{4}$, \\ Andree Rochfort ${ }^{5}$
}

${ }^{1}$ Director of Research, Irish College of General Practitioners, Dublin, Ireland; ${ }^{2} \mathrm{Head}$ Librarian, Irish College of General Practitioners, Dublin, Ireland; ${ }^{3}$ Librarian, Irish College of General Practitioners, Dublin, Ireland; ${ }^{4}$ Research Assistant, Irish College of General Practitioners, Dublin, Ireland; ${ }^{5}$ Director, Quality Improvement \& Doctors Health Programme, Irish College of General Practitioners, Dublin, Ireland

*For correspondence: claire. collins@icgp.ie

Competing interest: The authors declare that no competing interests exist.

Received: 22 December 2020 Accepted: 15 February 2021

Published: 21 April 2021

(C)This article is Open Access: CC BY license (https://creativecommons.org/licenses/by/4.0/)

Author Keywords: selfmanagement, patient empowerment, patient participation, primary health care, chronic conditions

Copyright (C) 2021, The Authors; DOI:10.3399/BJGPO.2020.0186

\begin{abstract}
Background: Primary care has a vital role in supporting patient autonomy to enable people with longterm conditions to manage their own health and wellness. Evidence is needed on whether education and training of health professionals helps support patient self-management and improves outcomes. The authors' first systematic review included only two articles showing patient outcomes following health professional training for promoting patient self-management.
\end{abstract}

Aim: To present an updated review undertaken from September 2013 to August 2018.

Design \& setting: A systematic review was undertaken using the PRISMA guidelines, following the methodology of the first review and is outlined in the PROSPERO registered protocol.

Method: Six databases were searched - Cochrane Library, PubMed, ERIC, Embase, Cumulative Index to Nursing and Allied Health Literature (CINAHL), and PsycINFO — in addition to web searches, hand searches, and bibliographies for articles published from 1 September 2013 to 31 August 2018.

Results: The updated systematic review showed more evidence is now available with 18 articles in the 5-year period from the 4284 abstracts located. Twelve of these articles showed a difference between intervention and control groups. Of the 18 articles identified, 11 were assessed as having a low risk of bias and five overall were rated of weak quality. The educational interventions with health professionals spanned a range of techniques and modalities, and many incorporated multiple interventions including patient components. There may be a lack of adoption owing to several challenges, including that complex interventions may not be delivered as planned and are difficult to assess, and owing to patient engagement and the need for ongoing follow-up.

Conclusion: More high-quality research is needed on what methods work best, for which patients, and for what clinical conditions in the primary care setting. The practical implications of training healthcare professionals require specific attention. 


\section{How this fits in}

Despite the vast literature on patient self-management, evidence on the association between training of health professionals in patient self-management and measured health outcomes was rare before and up to 2 years after its incorporation into the World Organization of Family Doctors (WONCA) Europe definition of general practice. Since the authors' previous systematic review, more published evidence is available to review (September 2013 to August 2018), which suggests a benefit to patient health outcomes and behaviour following health professional education. Interventions that include multiple aspects, follow-up, and patient-centred components are more likely to be successful; however, the implications for delivery and uptake in primary care need to be considered.

\section{Introduction}

The World Health Organization (WHO) defines chronic conditions as those that encompass disability and disease that people 'live with' for extended periods of time. ${ }^{1}$ The Chronic Care Model ${ }^{2}$ is an internationally accepted model for the management of non-communicable diseases and specifies self-management support as a key component. The concept of patient empowerment for selfmanagement was introduced into the WONCA Europe definition of general practice in $2011 .{ }^{3}$ Patient empowerment is a core concept of patient-centred care ${ }^{3,4}$ - a widely called for concept ${ }^{5}$ - and has been shown to be central to the improvement of self-management programmes, ${ }^{6,7}$ as has the need to recognise the phases of transformation for individual patients. ${ }^{8}$

Some studies demonstrate the benefit of self-management support ${ }^{9-18}$ for people with chronic conditions; however, it is also reported that patients with chronic conditions tend not to respond as well to lifestyle interventions. ${ }^{19}$ Primary care has a key role in supporting patient autonomy to enable patients to develop expertise in managing their own health and wellness. ${ }^{20}$ This support has been identified as a potentially impactful avenue, ${ }^{21}$ with education and training noted as potential ways of engaging primary care clinicians in patient self-management support. ${ }^{22}$ However, it is also recognised that visits in primary care may be brief and that low levels of readiness to change may exist among patients. ${ }^{23}$

The authors' first systematic review of 7533 abstracts published before September 2013 included only two articles showing patient outcomes following health professional training for promoting patient self-management. ${ }^{24}$ Both included articles suggested that primary care health professionals can help to harness patients' capacity to contribute to improvement of their own health outcomes. However, the review concluded the evidence was very limited on measured patient health outcomes.

The central focus of this project was to update that review and to systematically review the evidence from September 2013 to August 2018.

\section{Method}

A systematic review was undertaken using the PRISMA guidelines ${ }^{25}$ and follows the methodology outlined in the PROSPERO registered protocol. ${ }^{26}$

\section{Sourcing information}

Two specialist subject librarians assisted in the development of the search strategy, which replicated the strategy used in the first review and was designed to identify internationally recognised terminology in peer-reviewed journals. Full details of this strategy are available in the published protocol. ${ }^{26} \mathrm{Six}$ databases were searched - Cochrane Library, PubMed, ERIC, Embase, CINAHL, and PsycINFO — in addition to web searches, hand searches, and bibliographies. Articles published from 1 September 2013 to 31 August 2018 were included in the review, with the search conducted by two authors. The full search terms have been previously published. ${ }^{24}$

\section{Selection criteria}

Studies with the following designs were included: systematic reviews, meta-analysis, randomised controlled trials (RCTs), controlled clinical trials, interrupted time series, and controlled before-andafter studies. Participants were physicians in primary care settings, other clinicians in primary care settings, and patients aged $\geq 18$ years with chronic conditions in primary care settings. Included 
Table 1 Inclusion and exclusion criteria

\begin{tabular}{|c|c|c|}
\hline Inclusion criteria & Exclusion criteria & Exclusion code \\
\hline English articles & Non-English articles & Eng \\
\hline Adults (aged $\geq 18$ years) & Study population aged $<18$ years & Age \\
\hline Primary care or community & Secondary care or hospital & Not PC \\
\hline $\begin{array}{l}\text { Chronic conditions, chronic illness, chronic disease, } \\
\text { non-communicable disease (NCD) }\end{array}$ & Acute conditions & Acute \\
\hline $\begin{array}{l}\text { Study type: systematic reviews, meta-analysis, RCTs, } \\
\text { controlled clinical trials, interrupted time series, } \\
\text { controlled before-and-after studies }\end{array}$ & $\begin{array}{l}\text { Study type: qualitative studies, populations } \\
\text { studies, surveys, cross sectional, uncontrolled } \\
\text { before-and-after studies (cohort) }\end{array}$ & Study \\
\hline \multirow[t]{4}{*}{$\begin{array}{l}\text { Education and training of primary care health } \\
\text { professionals for patient education in promoting } \\
\text { change, behaviour change, lifestyle change, patient } \\
\text { engagement, patient empowerment, motivational } \\
\text { skills, patient collaboration, patient adherence and } \\
\text { compliance, patient self-management, decision } \\
\text { making, and patient problem-solving }\end{array}$} & $\begin{array}{l}\text { Not education or training of healthcare } \\
\text { professionals }\end{array}$ & lnt \\
\hline & Not primary care health professionals & Pop \\
\hline & Primary outcome measures not included & Out \\
\hline & Direct patient education only & Edu \\
\hline $\begin{array}{l}\text { Continuing education, CME, lifelong learning, or } \\
\text { evidence-based medicine }\end{array}$ & $\begin{array}{l}\text { Guideline adherence, clinical performance (no } \\
\text { educational component involved) }\end{array}$ & Guid \\
\hline \multirow[t]{4}{*}{$\begin{array}{l}\text { All studies published from } 1 \text { September } 2013 \text { to } 31 \\
\text { August } 2018\end{array}$} & Any article outside this timeframe & Date \\
\hline & Organisational interventions & Org \\
\hline & Financial changes and incentives & $\mathrm{Fi}$ \\
\hline & Regulatory interventions & Reg \\
\hline
\end{tabular}

$\mathrm{CME}=$ continuing medical education. $\mathrm{PC}=$ primary care. $\mathrm{RCTs}=$ randomised controlled trials.

interventions had an educational focus designed to train primary care clinicians to support patient selfmanagement. This review was concerned with all chronic conditions as they occur generically in the primary care setting, rather than focusing on any one specific chronic condition. Only articles including reference to patient outcomes, measured using validated measurement scales, were included. The primary patient outcome was change in patients' self-management behaviours. The secondary outcomes were changes in physical health measures; health behaviours, including medical adherence and compliance; service utilisation; psychological health; psychosocial function, for example, quality of life; physical functioning; and knowledge. The eligibility of studies was determined using the inclusion and exclusion criteria listed in the registered protocol and shown in Table 1.

\section{Data extraction}

All abstracts were reviewed using the RefWorks package to categorise the abstracts identified by the search. The initial review of abstracts was undertaken by one author, with $10 \%$ of abstracts re-checked by two other authors. The full-text articles of all those considered to be of possible relevance to the systematic review were read independently by two authors, and categorised using the same exclusion reasons. Disagreements were reviewed by another author. The quality assessment and extraction of thematic content of the final list of articles applicable to the systematic review question were considered by the two authors who read the full-text articles.

\section{Risk of bias and quality assessment}

The risk of bias was assessed using the Cochrane Collaboration's tool for randomised trials. ${ }^{27}$ It assessed the overall quality of individual studies using the Quality of Assessment Tool for Quantitative Studies. ${ }^{28}$ The risk of bias tool covers six domains of bias (selection bias, performance bias, detection bias, attrition bias, reporting bias, and other bias) with assessments on one or more aspects within 


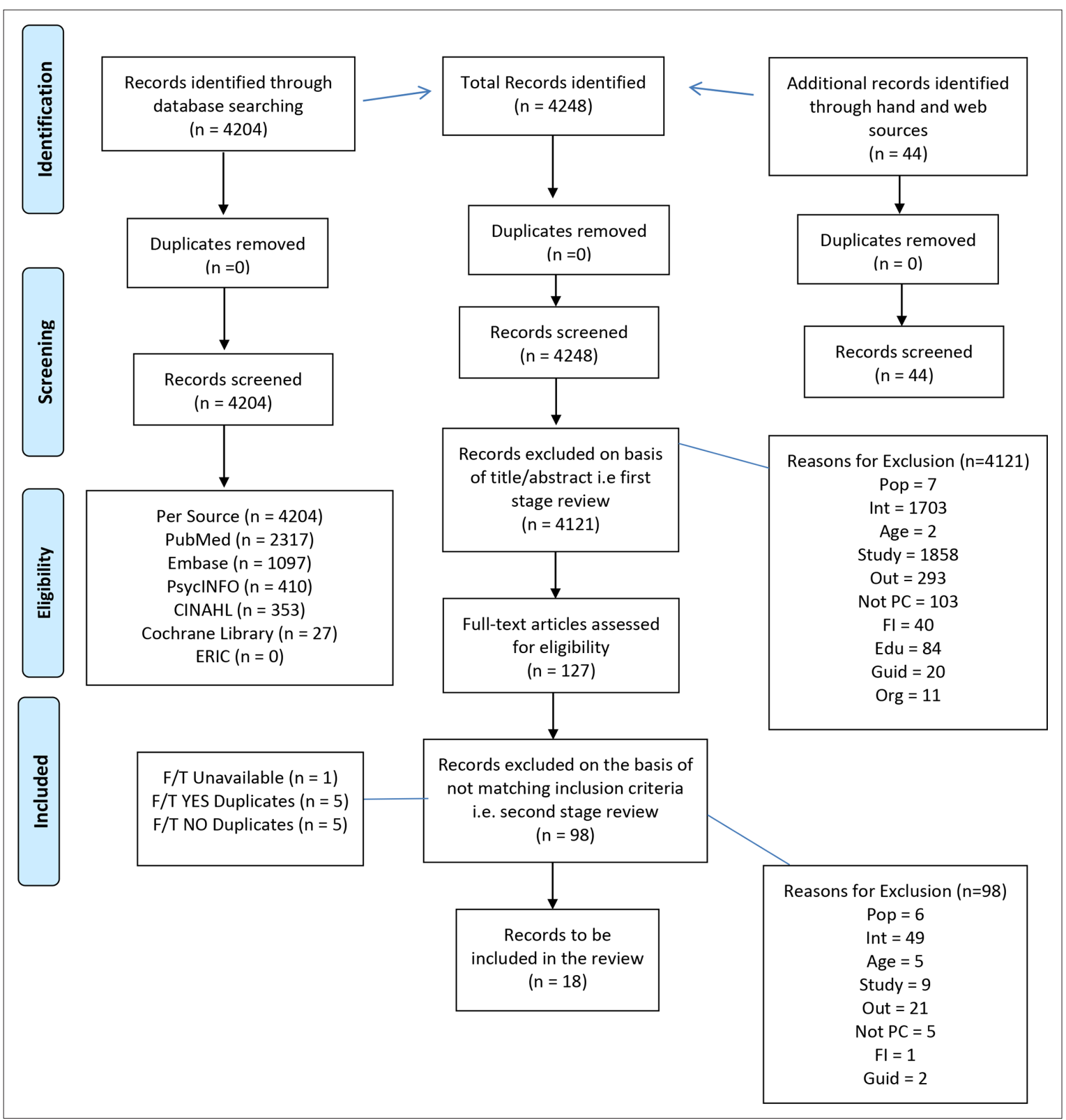

Figure 1 PRISMA flow diagram (see Table 1 for definition of exclusion code). F/T = full-text.

each. ${ }^{27}$ Reviewers rated six components of quality (selection bias, study design, confounders, blinding, data collection methods, and withdrawals and dropouts) leading to an overall methodological quality rating for each study of strong (no weak ratings), moderate (one weak rating), or weak (two or more weak ratings). ${ }^{28}$ Reviewers resolved rating disagreements through discussion.

\section{Data synthesis}

A narrative data synthesis was performed as per the authors' original protocol ${ }^{26}$ and the first systematic review ${ }^{24}$ on this topic.

\section{Results}

\section{Study review and selection}


Table 2 Quality rating of included papers

\begin{tabular}{|c|c|c|c|c|c|c|c|}
\hline First author & $\begin{array}{l}\text { Global } \\
\text { Score }\end{array}$ & $\begin{array}{l}\text { Selection } \\
\text { bias }\end{array}$ & $\begin{array}{l}\text { Study } \\
\text { design }\end{array}$ & $\begin{array}{l}\text { Confound- } \\
\text { ers }\end{array}$ & Blinding & $\begin{array}{l}\text { Data } \\
\text { collection } \\
\text { methods }\end{array}$ & $\begin{array}{l}\text { Withdraw } \\
\text { als and } \\
\text { drop outs }\end{array}$ \\
\hline Tobe $^{29}$ & weak & weak & $\bmod$ & weak & weak & strong & mod \\
\hline Kruis $^{30}$ & $\bmod$ & weak & strong & strong & $\bmod$ & strong & mod \\
\hline Vicens $^{31}$ & strong & strong & strong & strong & weak & strong & strong \\
\hline Keeley $^{32}$ & strong & mod & strong & strong & strong & strong & strong \\
\hline Kristoffersen ${ }^{33}$ & $\bmod$ & weak & strong & strong & strong & strong & strong \\
\hline van Dijk-de Vries ${ }^{34}$ & weak & weak & strong & weak & $\bmod$ & strong & $\bmod$ \\
\hline Racic $^{35}$ & strong & strong & strong & strong & $\bmod$ & strong & strong \\
\hline Vicens $^{36}$ & strong & strong & strong & strong & strong & strong & strong \\
\hline Kristoffersen ${ }^{37}$ & $\bmod$ & weak & strong & strong & strong & strong & strong \\
\hline$Z_{\text {war }}^{38}$ & weak & weak & strong & strong & strong & $\bmod$ & strong \\
\hline van Lieshout ${ }^{39}$ & $\bmod$ & weak & strong & strong & $\bmod$ & strong & strong \\
\hline Vaillant-Rousse ${ }^{40}$ & $\bmod$ & weak & strong & $\bmod$ & $\bmod$ & strong & $\bmod$ \\
\hline Griffiths $^{41}$ & strong & strong & strong & $\bmod$ & $\bmod$ & strong & $\bmod$ \\
\hline Eikelenboom ${ }^{42}$ & strong & strong & strong & strong & $\bmod$ & strong & strong \\
\hline Ramli43 & $\bmod$ & strong & strong & strong & weak & strong & strong \\
\hline Keeley ${ }^{44}$ & $\bmod$ & weak & strong & strong & $\bmod$ & strong & strong \\
\hline Kristoffersen ${ }^{45}$ & weak & weak & strong & strong & weak & strong & strong \\
\hline Baldeón ${ }^{46}$ & weak & weak & strong & weak & $\bmod$ & strong & $\bmod$ \\
\hline
\end{tabular}

$\bmod =$ moderate.

Overall, 4284 abstracts were found and 127 full-text articles were retrieved and read (Figure 1). Following the second-stage review, 18 articles reported patient outcomes and were included in the systematic review (see Supplementary Table S1).

All 18 articles were RCTs of educational interventions with primary care health professionals and examined their impact on patient outcome measures. ${ }^{29-46}$ The primary outcome of this review is the effectiveness of educational interventions with health professionals in terms of patient outcomes. Twelve of the 18 articles observed a significant difference between patient outcomes of those attending the intervention and control practices. ${ }^{29,31-33,35-37,41,43-46}$ Eleven articles overall - seven $31,33,36,37,41,43,44$ of the 12 articles showing an effect and four ${ }^{30,34,38,40}$ of the six articles not showing an effect - were considered to have a low risk of bias ${ }^{27}$ (see Supplementary Table S2). Among the seven articles that showed a difference in patient outcomes and had a low risk of bias, all were rated as moderate or strong in terms of the quality assessment (Table 2). ${ }^{28}$ Among the four trials that did not show significant differences in outcomes and were considered to have a low risk of bias, two were considered of weak quality and two of moderate quality.

All but two RCTs included condition homogeneous patients (those with diabetes, at risk of or with cardiovasular disease [CVD], asthma, chronic obstructive pulmonary disease [COPD], depression, or chronic headache). One study ${ }^{42}$ included patients with at least one chronic condition (diabetes, [risk of] CVD, asthma, or COPD) and one study ${ }^{31,36}$ included patients taking benzodiazepines daily for 6 months (including those with psychotic disorders, severe personality disorder, alcohol or illicit drug abuse, anxiety or depression in hospital, or being treated by a psychiatrist). Some studies reported multiple follow-up time points in one article, ${ }^{30,38,40,41,44}$ while other studies reported these in separate articles. ${ }^{31,33,36,37,45}$ Vicens et al reported on follow-up at 12 months and 16 months, ${ }^{31,36}$ and Kristoffersen et al reported at 3 months, 6 months, and an average of 16-months follow-up. ${ }^{33,37,45}$ Follow-up time varied across studies, from 1.5 months to 36 months among studies achieving differences between 
intervention and controls, and 3 months to 24 months among studies not showing differences in primary and/or secondary outcome measures.

The educational interventions with health professionals spanned a range of techniques and modalities, and many incorporated multiple interventions including patient components. None of the studies separated the impact of different intervention elements. Limited generalisability was a factor for all studies.

Successful programmes concluded that the need for ongoing patient follow-up and patient feedback is a time-consuming factor. ${ }^{31,36,44}$ However, a focus on person-centred care with individualised care plans and/or recording of lifestyle goals in the patient medical record were noted factors in some successful studies. ${ }^{29,31,36,41,46}$ One study surmised that a less time-consuming structured intervention with a written individualised stepped-dose reduction plan is as effective in primary care as a more complex intervention involving follow-up visits. ${ }^{31}$

There may be lack of adoption owing to several challenges, including that complex interventions may not be delivered as planned, ${ }^{31,32,36}$ often owing to workload implications, ${ }^{31,36,40}$ because of high dropout rates and low study integrity. ${ }^{38,40,42}$ Additionally, changes specifically owing to the interventions are sometimes difficult to assess. ${ }^{44}$ One study showed a positive impact of the intervention after 3-years follow-up to be 1.5 times more effective than usual care despite time and workload constraints. ${ }^{36}$ Booster training was included in some of the successful interventions. ${ }^{43,44}$ Cost-effectiveness analyses should form a part of all future evaluations according to one study, ${ }^{41}$ given the intensity of the interventions and evaluations required.

A focus on person-centred care where the care delivered is aligned to patients' needs and expectations and is interlinked to chronic disease management, increases the effectiveness of intervention programmes. ${ }^{46}$ Low uptake of some of the patient interventions, such as goal-setting and action-planning, and patient motivation were noted as factors that may have reduced impacts. ${ }^{30,38,41}$

Studies showing a positive intervention effect suggest that improvements can be maintained with strategies, such as ongoing patient follow-up, patient feedback, individualised care plans, recording of lifestyle goals in the patient medical record, and booster training. ${ }^{31,33,36,37,41,44,45}$

\section{Discussion}

\section{Summary}

The key finding of this systematic review is that since 2013, the scarcity of studies that assess the impact on patient outcomes of training primary care clinicians in patient self-management of chronic conditions has been somewhat addressed. However, the generalisability of results is limited and it is not clear which intervention aspects work best.

The updated systematic review showed more evidence is now available with 18 articles in 5 years from September 2013 to August 2018 from the 4284 abstracts located. Twelve of the 18 articles showed a difference between groups, indicating that training health professionals in general practice to support their patients' self-management activities results in improved patient outcomes. Seven of these were considered to have a low risk of bias, and overall nine were rated as moderate or strong on the quality assessment.

All educational interventions with health professionals in these articles spanned a range of techniques and modalities, and many incorporated multiple interventions including patient components. Several challenges, including that complex interventions may not be delivered as planned and are difficult to assess, ${ }^{31,32,36,44}$ often owing to workload implications, ${ }^{31,36,40}$ were found to be limiting factors. Patientcentred care appeared to increase the effectiveness of educational intervention with healthcare professionals in primary care. ${ }^{32,35,42,46}$ Some studies reported multiple follow-up time points in one article, ${ }^{30,38,40,41,44}$ while others reported these separately. ${ }^{31,33,36,37,45}$ Studies showing a positive intervention effect suggest that improvements can be maintained. ${ }^{31,33,36,37,41,44,45}$

\section{Strengths and limitations}

The systematic review was limited to articles where educational interventions for patient selfmanagement with health professionals in primary care were undertaken and the resultant patient outcomes were measured. Differences in terminology and concepts could have resulted in some articles not being located or included; however, the scope and criteria were clearly detailed. 
Only articles in English were included, which could lead to reporting and language bias. The quality of studies varied, which could have introduced biases that can lead to over- or under-estimation of intervention effectiveness. Seven of the 18 included articles did not follow intention-to-treat analysis, which could induce attrition bias.

\section{Comparison with existing literature}

Challenges to the delivery of such multifaceted programmes in primary care were identified by many of the studies. While some were related to research integrity, others were related to the feasibility of implementing interventions, particularly complex or prolonged interventions, in the real-world setting, as discussed elsewhere in the literature. ${ }^{23}$

Patient-centred care was identified as improving intervention effectiveness and is supported by findings that highlight the impact of good communication and trust, ${ }^{47,48}$ and the importance of personalised support and goal-setting, ${ }^{49}$ suggesting that empowerment-based strategies result in increased and longer self-efficacy improvement. ${ }^{7}$ This concept of patient-centred care supports the findings of the previous systematic review in relation to the role of motivational interviewing. ${ }^{24}$

\section{Implications for research and practice}

There is a need to distill what methods work best in different settings and for different patients. ${ }^{50,51}$ Incorporating the phases of transformation that individuals are in should be incorporated into future studies to enhance this understanding. ${ }^{8,23,52}$

Patient empowerment represents a challenge for healthcare professionals, ${ }^{7}$ and hence further research needs to ensure the contextual element is captured, ${ }^{8}$ and practice needs to find ways to overcome the real-world limits. ${ }^{7,23,48,51,53}$ Whole health system changes ${ }^{48,51,54}$ and the use of information and communication technology (ICT) are recommended. ${ }^{48,54}$

It has been recommended elsewhere, and is supported here, that treatment integrity and fidelity data should be reported in all behaviour change studies. ${ }^{23,53}$

Patient self-management support is recognised to be an effective component of comprehensive integrated chronic disease management. However, more high-quality research is needed on what methods work best, for which patients, and for what clinical conditions in the primary care setting. The practical implications of training healthcare professionals require specific attention.

Funding

None.

Ethical approval

Not required.

Registration

PROSPERO registered protocol: CRD42013004418.

\section{References}

1. World Health Organization. Innovative care for chronic diseases: building blocks for action: global report. 2002; https://www.who.int/chp/knowledge/publications/icccglobalreport.pdf (accessed 31 Mar 2021).

2. Wagner EH, Austin BT, Von Korff M. Organizing care for patients with chronic illness. Milbank Q 1996; 74(4): 511-544. DOI: https://doi.org/10.2307/3350391

3. WONCA Europe. The European definition of general practice/family medicine. 2011; https://www. globalfamilydoctor.com/site/DefaultSite/filesystem/documents/regionDocs/European\%20Definition\%20of\% 20general\%20practice\%203rd\%20ed\%202011.pdf (accessed 31 Mar 2021).

4. Mola E, De Bonis JA, Giancane R. Integrating patient empowerment as an essential characteristic of the discipline of general practice/family medicine. Eur J Gen Pract 2008; 14(2): 89-94. DOI: https://doi.org/10.1080/ 13814780802423463

5. Wagner EH, Austin BT, Davis C, et al. Improving chronic illness care: translating evidence into action. Health Aff 2001; 20(6): 64-78. DOI: https://doi.org/10.1377/hlthaff.20.6.64

6. Garvey J, Connolly D, Boland F, Smith SM. OPTIMAL, an occupational therapy led self-management support programme for people with multimorbidity in primary care: a randomized controlled trial. BMC Fam Pract 2015; 16: 59. DOI: https://doi.org/10.1186/s12875-015-0267-0 
7. Kuo C-C, Lin C-C, Tsai F-M. Effectiveness of empowerment-based self-management interventions on patients with chronic metabolic diseases: a systematic review and meta-analysis. Worldviews Evid Based Nurs 2014; 11(5): 301-315. DOI: https://doi.org/10.1111/wvn.12066

8. Yardley S, Cottrell E, Rees E, Protheroe J. Modelling successful primary care for multimorbidity: a realist synthesis of successes and failures in concurrent learning and healthcare delivery. BMC Fam Pract 2015; 16: 23. DOI: https:// doi.org/10.1186/s12875-015-0234-9

9. Bradley PM, Lindsay B, Fleeman N. Care delivery and self-management strategies for adults with epilepsy. Cochrane Database Syst Rev 2008; 2(2): CD006244.

10. Deakin T, McShane CE, Cade JE, Williams RDRR. Group based training for self-management strategies in people with type 2 diabetes mellitus. Cochrane Database Syst Rev 2005; 2: CD003417. DOI: https://doi.org/10.1002/ 14651858.CD003417.pub2

11. Holman H, Lorig K. Patient self-management: a key to effectiveness and efficiency in care of chronic disease. Public Health Rep 2004; 119(3): 239-243. DOI: https://doi.org/10.1016/j.phr.2004.04.002

12. Ofman JJ, Badamgarav E, Henning JM, et al. Does disease management improve clinical and economic outcomes in patients with chronic diseases? A systematic review. Am J Med 2004; 117(3): 182-192. DOI: https://doi.org/10. 1016/j.amjmed.2004.03.018

13. Marks R, Allegrante JP, Lorig K. A review and synthesis of research evidence for self-efficacy-enhancing interventions for reducing chronic disability: implications for health education practice (part I). Health Promot Pract 2005; 6(1): 37-43. DOI: https://doi.org/10.1177/1524839904266790

14. Du S, Yuan C. Evaluation of patient self-management outcomes in health care: a systematic review. Int Nurs Rev 2010; 57(2): 159-167. DOI: https://doi.org/10.1111/j.1466-7657.2009.00794.x

15. Lorig KR, Sobel DS, Stewart AL, et al. Evidence suggesting that a chronic disease self-management program can improve health status while reducing hospitalization: a randomized trial. Med Care 1999; 37(1): 5-14. DOI: https:// doi.org/10.1097/00005650-199901000-00003

16. Bodenheimer T, Wagner EH, Grumbach K. Improving primary care for patients with chronic illness. JAMA 2002; 288(14): 1775-1779. DOI: https://doi.org/10.1001/jama.288.14.1775

17. Becker A, Leonhardt C, Kochen MM, et al. Effects of two guideline implementation strategies on patient outcomes in primary care: a cluster randomized controlled trial. Spine 2008; 33(5): 473-480. DOI: https://doi.org/10.1097/ BRS.0b013e3181657e0d

18. Rubak S, Sandbaek A, Lauritzen T, et al. General practitioners trained in motivational interviewing can positively affect the attitude to behaviour change in people with type 2 diabetes. One year follow-up of an RCT, ADDITION Denmark. Scand J Prim Health Care 2009; 27(3): 172-179. DOI: https://doi.org/10.1080/ 02813430903072876

19. Ewenighi $\mathrm{CO}$, Uchechukwu $\mathrm{D}$, Adejumo BI, et al. Responses to glycemic control therapy according to age, gender, level of adiposity, and duration of diabetes in type 2 diabetic patients. Indian J Med Sci 2013; 67(3-4): 61-69. DOI: https://doi.org/10.4103/0019-5359.121117

20. Grüninger U, Kissling B. [Family medicine — a specialty of its own]. [Article in German]. Primary Care 2005; 5(11): 269-271.

21. Emmons KM, Rollnick S. Motivational interviewing in health care settings. Opportunities and limitations. Am J Prev Med 2001; 20(1): 68-74. DOI: https://doi.org/10.1016/S0749-3797(00)00254-3

22. Dennis C-L. Peer support within a health care context: a concept analysis. Int J Nurs Stud 2003; 40(3): 321-332. DOI: https://doi.org/10.1016/S0020-7489(02)00092-5

23. Keeley R, Engel $M$, Reed $A$, et al. Toward an emerging role for motivational interviewing in primary care. Curr Psychiatry Rep 2018; 20(6): 41. DOI: https://doi.org/10.1007/s11920-018-0901-3

24. Rochfort A, Beirne S, Doran G, et al. Does patient self-management education of primary care professionals improve patient outcomes: a systematic review. BMC Fam Pract 2018; 19(1): 163. DOI: https://doi.org/10.1186/ s12875-018-0847-x

25. Moher D, Liberati A, Tetzlaff J, et al. Preferred reporting items for systematic reviews and meta-analyses: the PRISMA statement. Ann Intern Med 2009; 151(4): 264-269. DOI: https://doi.org/10.7326/0003-4819-151-4200908180-00135

26. Collins $\mathrm{C}$, Rochfort $\mathrm{A}$, Beirne $\mathrm{S}$, et al. The effectiveness of educational interventions for primary care health professionals designed to improve self-management in patients with chronic conditions. PROSPERO: CRD42013004418. 2013; https://www.crd.york.ac.uk/prospero/display_record.php?ID=CRD42013004418 (accessed 31 Mar 2021).

27. Higgins JPT, Altman DG, Gøtzsche PC, et al. The Cochrane Collaboration's tool for assessing risk of bias in randomised trials. BMJ 2011; 343: d5928. DOI: https://doi.org/10.1136/bmj.d5928

28. Effective Public Health Practice Project. Quality assessment tool for quantitative studies; https://merst.ca/wpcontent/uploads/2018/02/quality-assessment-tool_2010.pdf (accessed 31 Mar 2021).

29. Tobe SW, Moy Lum-Kwong M, Von Sychowski S, et al. Hypertension management initiative prospective cohort study: comparison between immediate and delayed intervention groups. J Hum Hypertens 2014; 28(1): 44-50. DOI: https://doi.org/10.1038/jhh.2013.48

30. Kruis AL, Boland MRS, Assendelft WJJ, et al. Effectiveness of integrated disease management for primary care chronic obstructive pulmonary disease patients: results of cluster randomised trial. BMJ 2014; 349: g5392. DOI: https://doi.org/10.1136/bmj.g5392

31. Vicens $C$, Bejarano $F$, Sempere $E$, et al. Comparative efficacy of two interventions to discontinue long-term benzodiazepine use: cluster randomised controlled trial in primary care. Br J Psychiatry 2014; 204(6): 471-479. DOI: https://doi.org/10.1192/bjp.bp.113.134650 
32. Keeley RD, Burke BL, Brody $\mathrm{D}$, et al. Training to use motivational interviewing techniques for depression: a cluster randomized trial. J Am Board Fam Med 2014; 27(5): 621-636. DOI: https://doi.org/10.3122/jabfm.2014.05.130324

33. Kristoffersen ES, Straand J, Vetvik KG, et al. Brief intervention for medication-overuse headache in primary care. The BIMOH study: a double-blind pragmatic cluster randomised parallel controlled trial. J Neurol Neurosurg Psychiatry 2015; 86(5): 505-512. DOI: https://doi.org/10.1136/jnnp-2014-308548

34. van Dijk-de Vries A, van Bokhoven MA, Winkens B, et al. Lessons learnt from a cluster-randomised trial evaluating the effectiveness of self-management support (SMS) delivered by practice nurses in routine diabetes care. BMJ Open 2015; 5(6): e007014. DOI: https://doi.org/10.1136/bmjopen-2014-007014

35. Racic $\mathrm{M}$, Katic $\mathrm{B}$, Joksimovic $\mathrm{BN}$, et al. Impact of motivational interviewing on treatment outcomes in patients with diabetes type 2: a randomized controlled trial. J Fam Med 2015; 2(1): 1020. DOI: https://doi.org/10.1016/j.ijnurstu. 2011.11.011

36. Vicens $C$, Sempere $E$, Bejarano $F$, et al. Efficacy of two interventions on the discontinuation of benzodiazepines in long-term users: 36-month follow-up of a cluster randomised trial in primary care. Br J Gen Pract 2016; 66(643): e85-e91. DOI: https://doi.org/10.3399/bjgp16X683485

37. Kristoffersen ES, Straand J, Vetvik KG, et al. Brief intervention by general practitioners for medication-overuse headache, follow-up after 6 months: a pragmatic cluster-randomised controlled trial. J Neurol 2016; 263(2): 344-353. DOI: https://doi.org/10.1007/s00415-015-7975-1

38. Zwar NA, Bunker JM, Reddel HK, et al. Early intervention for chronic obstructive pulmonary disease by practice nurse and GP teams: a cluster randomized trial. Fam Pract 2016; 33(6): 663-670. DOI: https://doi.org/10.1093/ fampra/cmw077

39. van Lieshout J, Huntink E, Koetsenruijter J, Wensing M. Tailored implementation of cardiovascular risk management in general practice: a cluster randomized trial. Implement Sci 2016; 11: 115. DOI: https://doi.org/10.1186/s13012016-0460-0

40. Vaillant-Roussel $\mathrm{H}$, Laporte $\mathrm{C}$, Pereira $\mathrm{B}$, et al. Impact of patient education on chronic heart failure in primary care (ETIC): a cluster randomised trial. BMC Fam Pract 2016; 17(1): 80. DOI: https://doi.org/10.1186/s12875-016-0473-4

41. Griffiths C, Bremner S, Islam K, et al. Effect of an education programme for South Asians with asthma and their clinicians: a cluster randomised controlled trial (OEDIPUS). PLoS One 2016; 11(12): e0158783. DOI: https://doi.org/ 10.1371/journal.pone.0158783

42. Eikelenboom N, van Lieshout $\mathrm{J}$, Jacobs $\mathrm{A}$, et al. Effectiveness of personalised support for self-management in primary care: a cluster randomised controlled trial. Br J Gen Pract 2016; 66(646): e354-e361. DOI: https://doi.org/ 10.3399/bjgp16X684985

43. Ramli AS, Selvarajah S, Daud MH, et al. Effectiveness of the EMPOWER-PAR intervention in improving clinical outcomes of type 2 diabetes mellitus in primary care: a pragmatic cluster randomised controlled trial. BMC Fam Pract 2016; 17(1): 157. DOI: https://doi.org/10.1186/s12875-016-0557-1

44. Keeley RD, Brody DS, Engel M, et al. Motivational interviewing improves depression outcome in primary care: a cluster randomized trial. J Consult Clin Psychol 2016; 84(11): 993-1007. DOI: https://doi.org/10.1037/ccp0000124

45. Kristoffersen ES, Straand J, Russell MB, Lundqvist C. Lasting improvement of medication-overuse headache after brief intervention - a long-term follow-up in primary care. Eur J Neurol 2017; 24(7): 883-891. DOI: https://doi.org/ 10.1111/ene.13318

46. Baldeón ME, Fornasini M, Flores $\mathrm{N}$, et al. Impact of training primary care physicians in behavioral counseling to reduce cardiovascular disease risk factors in Ecuador. Rev Panam Salud Publica 2018; 42: e139. DOI: https://doi. org/10.26633/RPSP.2018.139

47. Legido-Quigley H, Camacho Lopez PA, Balabanova D, et al. Patients' knowledge, attitudes, behaviour and health care experiences on the prevention, detection, management and control of hypertension in Colombia: a qualitative study. PLoS One 2015; 10(4): e0122112. DOI: https://doi.org/10.1371/journal.pone.0122112

48. Gallardo-Rincón H, Saucedo-Martínez R, Mujica-Rosales R, et al. Online continuing medical education as a key link for successful noncommunicable disease self-management: the CASALUDTM model. Diabetes Metab Syndr Obes 2017; 10: 443-455. DOI: https://doi.org/10.2147/DMSO.S137891

49. Lenzen SA, Daniëls R, van Bokhoven MA, et al. Setting goals in chronic care: shared decision making as selfmanagement support by the family physician. Eur J Gen Pract 2015; 21(2): 1-7. DOI: https://doi.org/10.3109/ 13814788.2014.973844

50. Grol R. Improving the quality of medical care: building bridges among professional pride, payer profit, and patient satisfaction. JAMA 2001; 286(20): 2578-2585. DOI: https://doi.org/10.1001/jama.286.20.2578

51. Reynolds R, Dennis S, Hasan I, et al. A systematic review of chronic disease management interventions in primary care. BMC Fam Pract 2018; 19(1): 11. DOI: https://doi.org/10.1186/s12875-017-0692-3

52. Fisher L, Polonsky WH, Hessler D, Potter MB. A practical framework for encouraging and supporting positive behaviour change in diabetes. Diabet Med 2017; 34(12): 1658-1666. DOI: https://doi.org/10.1111/dme.13414

53. Bellg AJ, Borrelli B, Resnick B, et al. Enhancing treatment fidelity in health behavior change studies: best practices and recommendations from the NIH Behavior Change Consortium. Health Psychol 2004; 23(5): 443-451. DOI: https://doi.org/10.1037/0278-6133.23.5.443

54. Mogre V, Scherpbier AJJA, Stevens F, et al. Realist synthesis of educational interventions to improve nutrition care competencies and delivery by doctors and other healthcare professionals. BMJ Open 2016; 6(10): e010084. DOI: https://doi.org/10.1136/bmjopen-2015-010084 